\title{
Vigilância da saúde: fundamentos, interfaces e tendências
}

\author{
Health surveillance: foundations, interfaces and tendencies
}

Antonio Luis Vicente Arreaza ${ }^{1}$

JoséCássio de M oraes ${ }^{2}$

${ }^{1}$ Instituto Adolfo Lutz, Coordenadoria deControle deDoenças, Secretaria de Estado da Saúdede São Paulo. Rua Silva Jardim 90, Vila Nova. $11015-020$ Santos SP.

alvarreaza@uol.com.br ${ }^{2}$ Departamento deM edicina Social, Faculdade de Ciências M édicas, Santa Casa de São Paulo.
Abstract The present article rescues initially the forms, content and operational projection of the epidemiological surveillance as indispensable tool for the knowledge field and public health practices. After that, we verify that the health surveillance model establishes an enlargement of this operational concept of surveillance by integrating the collectives and individuals practices in different health necessities dimensions, which includes beyond of the risks and damages control also theeco-socials determinants. In the sequence, we search to dimension the distinct levels of actuation of this sanitary practice articulated to the interventions of promotion, protection and recovery under a located and integrated logic of the unique system of Brazilian health. Finally, we argue that all the conceptual-operational model framework of publichealth surveillanceitself constitutes as a politics and sanitary base for the consolidation of the health promotion paradigm in the collective health field.

Key words Health surveillance, Sanitary practice, Public health
Resumo 0 presente artigo resgata inicialmente as formas, conteúdo e projeção operacional da vigilância epidemiológica como instrumento indispensável para o campo do saber epráticas da saúde pública. Em seguida, verificamos que o modelo de vigilância da saúde estabelece uma ampliação desse conceito operacional de vigilância, ao integrar as práticas coletivas e individuais em diferentes dimensões das necessidades de saúde, que incluem além do controle de riscos e danos, os determinantes ecossociais. $\mathrm{Na}$ sequência, buscamos dimensionar os distintos níveis de atuação desta prática sanitária articulados às intervenções de promoção, proteção e recuperação, sob uma lógica regionalizada e integralizada do Sistema Único de Saúde brasileiro. Por fim, argumentamos que todo o arcabouço conceitual-operacional de vigilância da saúde se constitui como uma base política e sanitária para a consolidação do paradigma da promoção da saúde no campo da Saúde Coletiva.

Palavras-chave Vigilância da saúde, Prática sanitária, Saúde coletiva 
Fundamentos

A trajetória conceitual

da vigilância em saúde pública

$O$ conceito de vigilância como um instrumento de saúde pública surgiu no final do século XIX com o desenvolvimento da microbiologia e de saberes sobre a transmissão de doenças infecciosas, processando dados para subsidiar o empre go de medidas oportunas às pessoas observadas como uma al ternativa à prática restritiva da quarentena. Esse conceito envolvia a manutenção do alerta responsável e a observação dos contatos de pacientes das pestilências; seu propósito fundamental era detectar doentes já em seus primeiros sintomas para a rápida instituição do isolamento. Em nosso país, os termos inicialmente utilizados com esse significado foram vigilância médica e, posteriormente, sanitária; esta, a sua vez, constituía em vigiar os comunicantes durante o período de incubação da enfermidade a partir da data do último contato com um doente ou do momento em que o comunicante abandonou o local em que se encontrava a fonte de infecção ${ }^{1}$.

No século XX, assistimos a expansão do conceito de vigilância e ao desenvolvimento de diferentes sistemas com métodos de coleta, análise e difusão de dados diversificados; contudo, somente a partir dos anos cinquenta, observamos uma modificação desse conceito, que passava a ter um sentido mais abrangente: 0 de acompanhamento sistemático da incidência e distribuição das doenças por meio da consolidação e avaliação dos registros de morbi-mortalidade como de outros dados relevantes para a saúde pública, cabendo a vigilância disseminar regularmente as informações a todos que fossem necessário². Nota-se que, nesta definição, a vigilância não envolveria a imediata responsabilidade pelas atividades de controle, constituindo-se mais no alerta e orientação à autoridade de saúde para que se pudesse tomar a decisão adequada ${ }^{3}$.

Um dos principais fatores que contribuíram para a larga utilização desse instrumento de saúde pública, em todo mundo, foi a campanha de erradicação da varíola nas décadas de sessenta e setenta. Temos então, especialmente nos países em desenvolvimento, a ampliação do conceito de vigilância, que passava a abranger também as medidas de controle. A partir dessa experiência, que se desenvolveu em inúmeros países, com diferentes tipos de estruturas organizacionais de sistemas de saúde, a vigilância passaria a ser apreendida como um sistema para a agilização das ações de controlee deinteligência epidemiológica para 0 aprimoramento das estratégias de intervenção executadas pelo setor saúde'1.

Foi nesse contexto que, na 21a Assembléia da OM S de 1968, a vigilância passou a ser concebida como uma função essencial em saúde pública, sendo definida de forma mais ampla sem uma distinção precisa entre a vigilância e a pesquisa científica: 0 estudo epidemiológico das doenças entendidas como um processo que envolvia a ecologia do agente etiológico, o hospedeiro, o reservatório e os vetores, e também os complexos mecanismos de disseminaç̧ão e extensão das infecções propagadas ${ }^{4}$. Foi então que se consolidou em nosso país o conceito de vigilância no controlede doenças transmissíveis, ficando a vigilância sanitária mais restrita ao conjunto de ações nas áreas de portos e fronteiras e passando a incluir, a partir dos anos setenta, o controle sanitário de produtos e serviços de interesse à saúde da soci edade ${ }^{5}$.

Esse processo de consolidação da vigilância, na mesma época, sedimentou uma distinção entrea vigi lância epidemiológica (VE) ea sanitária: a primeira orientada para o controle de casos e contatos obedecen do a uma racional idade técnico-sanitária fundamentada na clínica ena epidemiologia; já a segunda, dirigida para os ambientes, produtos e serviços obedecen do a uma racionalidade político-jurídica alicerçada na regulamentação da produção, distribuição e consumo de bens e serviços ${ }^{6}$.

Já nos anos oitenta, a vigilância passou a ser definida como a coleta, análise einterpretação dos dados acerca dos agravos específicos de saúde que afetam a população, estando integrada com a rápida disseminação das informações para todos os responsáveis pela prevenção e controle dos mesmos ${ }^{7,8}$, delimitando, enfim, essa prática epidemiológica específica em face à abrangência da utilização da epidemiologia nos serviços de saúde.

De qualquer modo, a consolidação da vigilância, na segunda metade do século passado, representou um avanço significativo na medida em que permitiu a incorporação da mesma nas atividades regulares dos serviços como um importante instrumento de saúde pública, sendo utilizado não só para doenças transmissíveis, como também para outros relevantes agravos à saúde, apresentando variações em sua abrangência em países com diferentes sistemas sociopolíticos e econômicos e com distintas estruturas de serviços de saúde?.

A partir dos anos noventa, diante da crise do setor saúde em nosso país, o movimento sanitário não se limitou a elaborar propostas racionali- 
zadoras como a de vigilância em saúde pública, propondo, sim, mudanças em direção à democratização das práticas de saúde na perspectiva do Sistema Ú nico de Saúde(SUS). Isto implicou a própria reorganização das ações de vigilância epidemiológica e sanitária para a redução de riscose danos das doenças, como a identificação dos determinantes da saúde coletiva e individual ${ }^{10}$.

A necessidade de reorganizar o setor saúde em face aos princípios de universalização, integralidade e equidade na prestação de serviços le vou os agentes de saúde pública a buscar na epidemiologia respostas para as várias questões emergentes no processo de construção do SUS. Nesse movimento de mudanças, os epidemiologistas procuraram afirmar a priori que as contribuições desse saber não deveriam ficar restritas às tradicionais atividades de VE equea sua racionalidade encerraria possibilidades mais amplas de atuação, não se restringindo ao fornecimento deelementostécnico-científicos para um conjunto limitado de agravos à saúde. Partindo então do pressuposto de que os serviços deveriam organizar-se tendo por finalidade a satisfação das necessidades de saúde tal como se apresentam no plano coletivo e individual, o diagnóstico epidemiológico orientaria desde sempre os planos de ação, delimitando os campos de atuação e as opções possíveis em saúde pública ${ }^{11}$.

No caso da vigilância como uma prática epidemiológica, entre os focos de sua atenção estariam não somente o estado de saúde da população, através do monitoramento das doenças e fatores de riscos, mas também as tecnologias desenvolvidas, o acesso equitativo, o processo de trabal ho ea utilização dos serviços com qualidade e efetividade ${ }^{12}$. Todas essas tendências, todavia, vêm se fortalecendo desde a contínua reorganização do sistema deVE no contexto do SUS, superando a dicotomia tradicional entre vigilância-assistência a partir das práticas de vigilância à saúde e pela incorporação das ações de vigilância em todas as unidades básica de saúde, inclusive nos hospitais da rede de referência ${ }^{13}$.

Já o modelo de vigilância da saúde (VS), ao enfatizar as condições de vida, estabelece a sua vez uma ampliação desse conceito operacional de $V E$, integrando as práticas coletivas e individuais em diferentes dimensões das necessidades sociais de saúde, que incluem além do controle de riscos, danos e agravos, os seus determinantes ecossociais, subsidiando amplamente a gerência no processo de definição dos problemas prioritários e das possíveis respostas sociais para enfrentá- $\operatorname{los}^{14}$.
O objeto-problema de preocupação ampliase para além dos fatores de risco ou doenças e doentes, passando a incidir sobre as necessidades e determinantes dos modos de vida e saúde. Os meios de trabalho também se ampliam, incorporando à tecnologia médico-sanitária o planejamento, a programação e avaliação do impacto das ações sobre o perfil epidemiológico da população. Por fim, as formas de organização dos processos de trabal ho transcendem os espaços convencionais de saúde, estendendo-se para outros setores públicos e não públicos comprometidos com as políticas saudáveis e ações intersetoriais ${ }^{10}$.

A implementação desta prática sanitária implicaria, portanto, a recomposição dos meios de trabalho para a operacionalização desse objetoreal, a reestruturação das atividades dos seus agentes e sujeitos ea redefini ção das relações técnico-sociais das organizações de saúde sob as quais se realiza o processo de trabalho produtivo. Cada uma dessas modificações pode ser provocada pela identificação denovos problemas ou necessidades de saúde, como mediante a elaboração de conceituações distintas acerca do objeto dessas práticas ${ }^{10}$.

Esse modo tecnológico de intervenção seria então uma alternativa em defesa da saúde e meIhoria das condições de vida, constituindo-se de fato numa estratégia de organização de um conjunto de políticas epráticas, queassumiriam configurações específicas conformea situação de saúde das diferentes coletividades. Ao entender 0 problema como um impedimento à plena realização dos modos de andar a vida, ou ainda uma ameaça concreta ao bem-estar social das pessoas, poderia ajudar a saúde, fortalecendo a emancipação dos sujeitos e intervindo sobre situações e não unicamente sobre riscos ${ }^{15}$.

Posto isso, este artigo resgata inicialmente as formas, conteúdo e projeção operacional da VE como instrumento indispensável para o campo do saber e práticas de saúde pública. Em seguida, verificamos que o modelo de vigilância da saúde estabelece uma ampliação desse conceito operacional de vigilância ao integrar as práticas coletivas e individuais em diferentes dimensões das necessi dades sociais de saúde, que incluem além do controle de riscos e danos também os determinantes ecossociais. $\mathrm{Na}$ sequência, buscamos dimensionar os distintos níveis de atuação desta prática sanitária articulados às intervenções de promoção, prevenção e recuperação sob uma lógica regionalizada e integralizada do SUS. Por fim, argumentamos que todo o arcabouço con- 
ceitual-operacional de vigilância da saúde seconstitui como uma base política e sanitária para a consolidação do paradigma da promoção da saúde no campo da Saúde Coletiva.

A prática epidemiológica

no âmbito da saúde pública brasileira

As ações de controlee preven ção das doenças transmissíveis baseiam-se em intervenções que atuam sobre as conexões conhecidas da cadeia epidemiológica, sendo capazes de vir a interrompêla oportunamente. Contudo, a interação entre o homem e 0 ambiente é bastante complexa, envolvendo determinantesnão totalmenteconhecidos ou que podem se modificar no momento em que se desencadeiam as ações desaúde pública. Sendo que as estratégias de intervenção tendem a ser aprimoradas ou substituídas na medida em que novos saberes são aportados, seja por pesqui sas científicas ou pela observação sistemática da dinâmica dos métodos de prevenção e controle estabelecidos. 0 desenvolvimento desses saberes contribui também para a modificação de conceitos e das formas organizacionais dos serviços de saúde numa busca contínua do seu aprimoramento e adequação no atendimento à população ${ }^{16}$.

A trajetória conceitual da vigilância epidemiológica e a transformação de sua prática, na segunda metade do século passado, podem ser compreendidas a partir dessa contextualização. Originalmente, essa expressão significava a observação sistemática e ativa de casos suspeitos ou detectados de doenças transmissíveis e deseus contatos. Tratava-se, portanto, da vigilância de pessoas por meio de medidas de isolamento ou de quarentena aplicadas individualmente e não de forma coletiva. Posteriormente, na vigência das campanhas de erradicação de doenças, a vigilância epidemiológica passou a ser referida como uma das etapas destes programas, na qual se buscava detectar ativamente a existência de casos da doença-alvo, com vistas ao desencadeamento de medidas urgentes destinadas a bloquear a sua transmissão. A estrutura operacional devigilância organizada para esse fim deveria ser desativada após a comprovação de que o risco de transmissão fora eliminado como resultado das ações do programa.

No decorrer dos anos sessenta, consolidouse internacionalmente uma conceituação mais abrangentede vigilância epidemiológica, em que eram explicitados seus propósitos, funções, atividades e modalidades operacionais, sendo en- tão definida como o conjunto de atividades que permite reunir a informação indispensável para se conhecer, a qualquer momento, o comportamento ou a história natural das doenças, como detectar ou prever alterações dos seus fatores condicionantes, com a finalidade de recomendar as medidas apropriadas que levem à prevenção e ao controle de determinadas doenças transmissívei $\mathrm{s}^{16}$.

Em 1975, por recomendação da 5a Conferência Nacional de Saúde, foi instituído o sistema nacional devigilância epidemiológica (SNVE) que, a sua vez, formalizado e regulamentado, incorporou o conjunto de doenças transmissíveis até então consideradas de mai or relevância sanitária no país. Buscava-se na ocasião compatibilizar a operacionalização das estratégias de interven ção desenvolvidas para controlar os agravos específicos à saúde através de programas nacionais que eram na época escassamente interativos ${ }^{16}$.

O sistema tornava-seresponsável pelo controle dedoenças endêmicas que, anteriormente, eraatribuição dos vários serviços nacionais, como o de malária ou tuberculose. Adotando um modelo operacional horizontal no qual atuariam os três níveis distintos da estrutura dos serviços de saúde, foi destinado ao M inistério da Saúdeà competência de normatização e coordenação das atividades $^{17}$. Por conseguinte, amplia-se o leque das doenças sob notificação compulsória, incorporando, além das previstas pelo regulamento sanitário internacional, aquelas vinculadas ao programanacional de imunização, como as controláveis através de ações coordenadas por órgãos específicos da saúde e as meningites em geral ${ }^{18}$.

Com a promulgação da Lei $n$ ㅇ 8.080/90, 0 SNVE sofre mudanças importantes na medida em que as proposições sobre a reorganização do setor saúde se encontram ancoradas nas principais diretrizes do SUS, seja através da integralidade das ações de saúde, eliminando a dicotomia tradicional que tanto dificultava a atuação da VE, seja mediante a inclusão dos municípios na gestão da saúde alicerçada pelo processo de descentral ização do SUS. Isto provavelmente deveria suplantar a desvinculação administrativa entre assistência médica e saúde pública; entender-se-ia assim por integralidade da atenção à saúde como "um conjunto articulado de ações e serviços, preventivos e curativos, integrados numa rede regi onalizada e hierarquizada constituída num sistema único de saúde"19.

De acordo com essa Lei O rgânica da Saúde, a VE pôde então ser definida mantendo a essência das concepções que vinham sendo adotadas, mas já se inserindo no contexto do SUS, como um 
conjunto de ações que proporcionam o conhecimento, a detecção ou prevenção de qualquer mudança nos determinantes e condicionantes da saúde individual ou coletiva, com a finalidade de recomendar eadotar as medidas de prevenção e controle das doenças ou agravos à saúde ${ }^{16}$. Tal definição, além de não restringir a VE ao controle das doenças transmissíveis, propõe uma investigação mais orientada e integrada para os condicionantes do processo da saúde-doença entre os planos coletivo eindividual.

0 esquema de trabalho da vigilância estaria relacionado a priori à descrição dos padrões de ocorrência das doenças notificáveis, ao monitoramento de ten dências de agravos relevantes em saúde pública, como no alerta às pessoas suscetíveis a determinados agentes, vias e fontes de exposição, para os quais existam intervenções eficazes de prevenção, proteção e controle. De outro lado, destacamos a alocação apropriada de recursos, a priorização dos problemas de saúde, as bases técnicas dos programas de prevenção e controle, a avaliação das estratégias de intervenção, como do impacto dessas medidas na população-alvo ${ }^{1}$.

A vigilância como uma ferramenta de priorização das ações em saúde permite assim monitorar o estado de saúde da população em relação aos agravos que foram e são objeto de prevenção e controle. Trata-se de um conjunto de atividades destinadas a obter as informações necessárias para se conhecer a situação epidemiológica em questão, detectando ou prevendo as alterações prováveis dos condicionantes focalizados e orientando a aplicação de medidas preventivas, mantendoas ou modificando-as, com o propósito de atingir os melhores resultados possíveis em termos de efetividade e impacto na população ${ }^{20}$.

Nesse sentido, as informações produzidas oferecem subsídios importantes para estimar a magnitude dos problemas de saúde e aplicar oportunamentea prevenção apropriada, além de prognosticar a evolução do estado de saúde presentena col etividade. Isso deveria nortear as ações de controle e aquisição de saberes consistentes, visando melhorar os efeitos obtidos das medidas colocadas em prática no decurso das intervenções em saúde pública.

Em síntese, a vigilância tem como propósito fundamental fornecer a orientação técnica para os que respondem pela execução das ações de controle dos agravos e danos, tornando disponíveis, para este fim, as informações atualizadas sobre a ocorrência desses eventos como dos seus determinantes numa dada área geográfica ou po- pulação específica. Logo, a VE se constitui também num importante instrumento para o planejamento, organização e avaliação das intervenções, como para a normatização de atividades técnicas correlatas dos serviços de saúde ${ }^{21}$.

Assim, é compreendida como um processo dinâmico que desencadeia um conjunto deatividades ordenadas, complexas e hierarquizadas, podendo ser razoavelmentedelimitadas por meio de seus objetivos e metas específicas, do perfil profissional das equipes de saúde e da infraestrutura e tecnologias para a execução de suas tarefas, resultando num trabalho coletivo e integrado que se contextualiza no âmbito das práticas de saúde pública.

\section{Interfaces}

O modelo de vigilância da saúde

e suas interfaces político-sanitárias

A despeito do debate quevem sendo travado, desde o final dos anos oitenta, sobre a utilização da epidemiologia nos serviços de saúde, refere-se especialmente à análise das condições de vida e saúde de populações distintas com o propósito de alcançar uma maior efetividade na planificação e gestão do setor saúde, contribuindo assim para a identificação de prioridades e avaliação dos programas e serviços de saúde ${ }^{22}$. Já no âmbito da oferta organizada, espaço de articulação do enfoque epidemiológico, a programação e a execução das ações partiriam então da priorização dos problemas e necessidades de saúde em territórios delimitados, como na proposta de reorganização das práticas sanitárias no contexto de construção do SU S10,23.

Desse modo, a partir dos anos noventa, 0 emergentemodel o devigilância da saúdejá apontava para a superação da dicotomia dentre as ditas práticas coletivas e individuais por meio das contribuições do planejamento, epidemiologia e ciências sociais, tendo como suportepolítico-institucional o processo de descentralização e reorganização dos serviços e práticas de saúde em nível local ${ }^{10}$. Tal tendência já concebia a noção de vigilância à saúde como uma forma de incorporar o amplo papel da epidemiologia em suas propostas de ação e, ao abordar o tema da desigualdade social em saúde, significava um deslocamento conceitual do saber epidemiológico face ao exclusivo controle dos riscos e danos para a compreensão das relações sociais que determinam a distribuição desigual dos agravos à saúde ${ }^{24}$. 
$\mathrm{Na}$ mesma época, surge oportunamente a distinção entre uma concepção restrita e outra ampliada desse novo modelo assistencial. A primeira entendia por vigilância à saúde um conjunto de ações voltadas para o conhecimento, prevenção e enfrentamento contínuo dos problemas de saúde, e de seus fatores de riscos, como dos acidentes, incapacidades, doenças crônico-degenerativas e outros relevantes agravos de saúde ${ }^{25}$, significando uma ampliação da VE com a incorporação da sanitária sem, contudo, prever a reorganização dos serviços e ações da atenção integral à saúde, aí incluídas as intervenções sobre os determinantes sociais, de um lado, e a assistência médico-hospitalar, de outro ${ }^{10}$.

A concepção ampliada de vigilância da saúde resgataria, por sua vez, o desenvolvimento conceitual-metodológico que vinha se verificando a partir de uma visão abrangente de saúde e da formulação de model os conceituais das causas, riscos e danos, operacionalizando-os para o resgate e ampliação do modelo dehistória natural das doenças, incorporando desde as ações sociais organizadas até as intervenções sobre os riscos e agravos, bem como as de recuperação e reabilitação dos indivíduos enfermos ${ }^{25}$.

Em vista disto, a vigilância da saúde, como uma proposta de recomposição das práticas sanitárias, privilegiaria tanto sua dimensão técnica conformada por "um conjunto de ações em saúdequeencerram distintas combinações tecnológicas destinadas a controlar determinantes, riscose danos" ${ }^{\prime 2}$, quanto sua dimensão gerencial como "uma prática estratégica que organiza os processos de trabalho produtivo sob a forma de operações para confrontar os problemas e necessidades de saúde num dado território delimitado"26.

Com efeito, isto diz respeito à própria dimensão operacional das práticas de saúde na organização social da produção e prestação de bens e serviços, correspondendo aos modos de combinar tecnologias, materiaisenão materiais, utilizadas nos processos de trabal ho coletivo e resultando na oferta organizada e ações programáticas de saúde, significando um conjunto articulado e integrado de práticas destinadas ao controle decausas, riscos e danos à saúde, como na flexibilização da organização desse trabalho produtivo para 0 enfrentamento dos problemas priorizados nos diferentes tempos do processo saúde-doença. Todo esse referencial contempla, em última instância, a formulação de políticas públicas saudáveis eintervenção social organizada sobrea situação de saúde das coletividades nos seus distintos modos de andar a vida ${ }^{10,15}$ (Figura 1).
Ao se considerar a possibilidade de intervenções voltadas para o controle de riscos, danos e determinantes, pensava-se na perspectiva de incorporar a promoção e proteção da saúde ao conjunto articulado de medidas já adotadas pelo SUS. Logo, as ações programáticas e oferta organizada no setor saúde poderiam se articular com as atividades de vigilância sanitária e epidemiológica, como da assistência médico-hospitalar. Da mesma forma, tais ações setoriais seriam capazes de se integrar à intervenção social organizada e às políticas intersetoriais ou transetoriais que configuram o espaço da promoção da saúde. Se essas fossem efetivamente implementadas, tomando a saúde e a qualidade de vida como referentes fundamentais, a ação transetorial consequente estaria voltada para as políticas públicas coerentes com o movimento de promoção da saúde ${ }^{15,23}$ (Figura 2).

Todavia, ressaltamos que as práticas de VS também diferem das ações tradicionais de VE e sanitária ao apontarem para a inclusão de outros sujeitos, gerentes de serviços, técnicos e representantes de coletivos organizados para participar com seus diferentes pontos de vista no processo de trabalho coletivo da saúde. A vigilância da saúde propõe assim a incorporação de novos atores, extrapolando o conjunto de profissionais e trabalhadores da saúde ao envolver a coletividade organizada, o que corresponde, por sua vez, à ampliação do objeto-real para além dos fatores clínico-epidemiológicos no âmbito individual e coletivo, abarcando determinações que afetam os diferentes grupos sociais em função de suas condições de vida e saúde ${ }^{10}$.

N essa perspectiva, a intervenção dos seus meios de trabalho extrapola também o uso de tecnologias médico-sanitárias e inclui as de comunicação social e gestão ampliada em saúde, que motivam a mobilização e atuação dos diversos grupos de interesse na promoção e defesa dos modos de vida com saúde. Já as formas de organização do seu trabalho transcendem aos espaços institucionais dos serviços de saúde, se estendendo a outros setores e contextos sociopolíticos de ação do Estado e da sociedade civil, envolven do uma rede complexa de grupos deinteresse comprometidos com as políticas e práticas de promoção da saúde.

Adotar a concepção ampliada de VS, visando à transformação do modelo de atenção à saúde local, implica tanto em avançar no processo de descentralização da gestão e gerência da saúde nos territórios municipais, como investir na articulação transetorial, na reorganização da aten- 


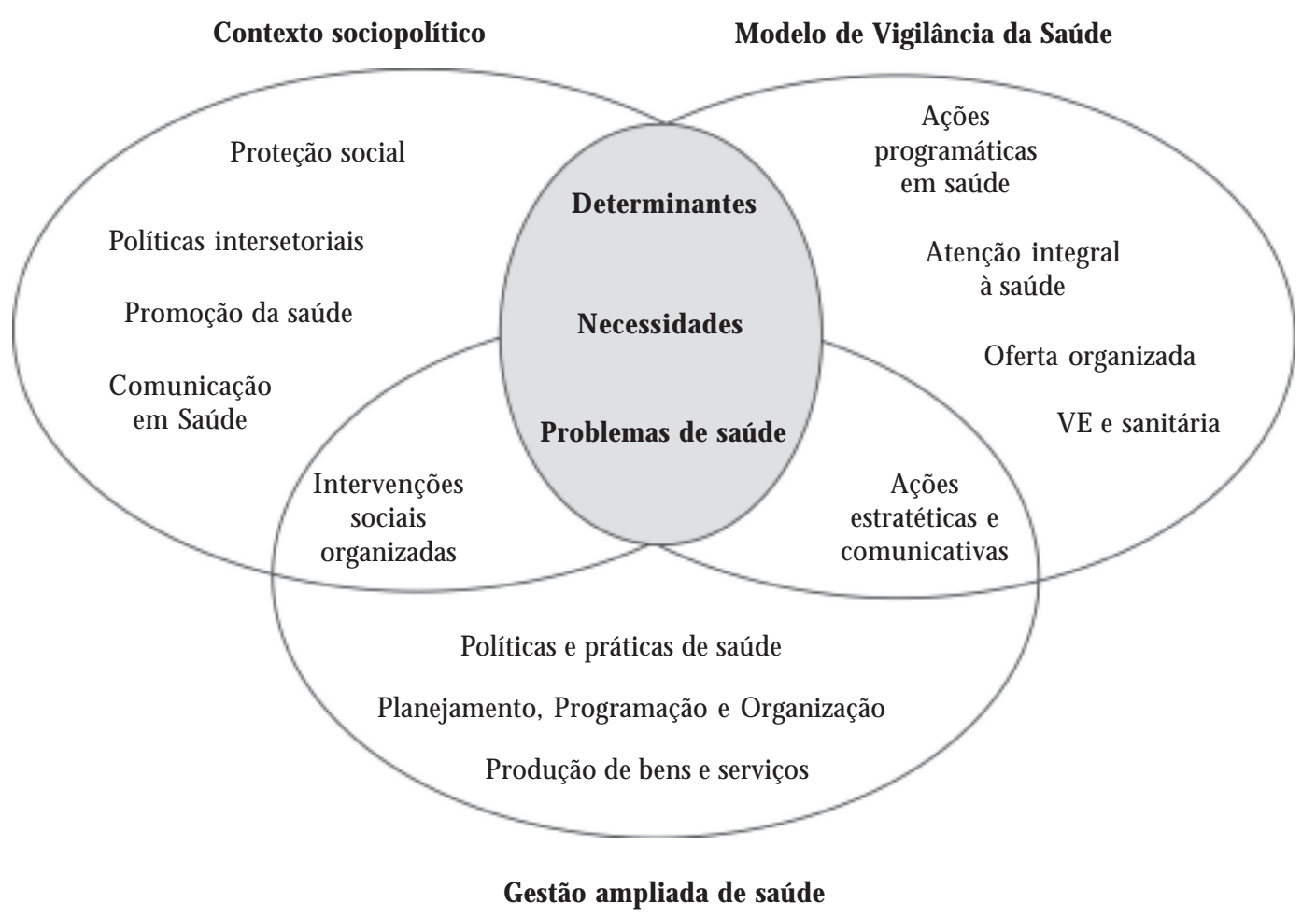

Figura 1. M odelo político-sanitário da saúde no contexto do Sistema Ú nico de Saúde.

Adaptação: Teixeira CF, Paim JS, Vilasbôas AL ${ }^{10}$.

ção básica e no fortalecimento do controle social sobre a gestão dos serviços de saúde do SUS. Ademais, a operacionalização das práticas de VS para o enfrentamento dos problemas e necessidades de saúde deve-se dar segundo uma lógica que privilegie não a organização de estruturas tecnocráticas, mais sim a flexibilização gerencial na reorientação das equipes de saúde, como a participação social no processo de implementação das ações ${ }^{10}$.

Implicando uma visão ecossocial ao processo saúde-doença, abre-se caminho para uma perspectiva de intersetorialidade e integralidade das ações em Saúde Coletiva, direcionando a reformulação dos modelos assistenciais constituídos por processos de trabal ho articulados em operações e integrando as ações estratégicas sobre os nós críticos dos problemas coletivos de saúde e de seus efeitos emergentes nos indivíduos e grupos sociais, em face de suas condições de vida em um dado território político, econômico, cultural eideológico ${ }^{27,28}$.

Em síntese, os modelos assistenciais podem ser considerados como um dado modo de combinar tecnologias para intervir sobre problemas e atender necessidades sociais de saúde, sendo uma maneira de organizar os meios de trabalho utilizados nas práticas ou programas de saúde. Sinalizam como melhor integrar os saberes einstrumentos disponíveis para resolver os problemas de saúde, incorporando uma lógica que orienta as práticas sobre as "demandas potenciais de saúde". Aliás, adotamos também a expressão utilizada por Paim como "modo tecnológico de intervenção em saúde" para designar o modelo de vigilância da saúde $e^{15}$.

Por fim, na recomposição das práticas sanitárias do SUS, o repensar a epidemiologia como meio de trabalho significa concebêla como uma "tecnologia não material", ou seja, uma ferra- 


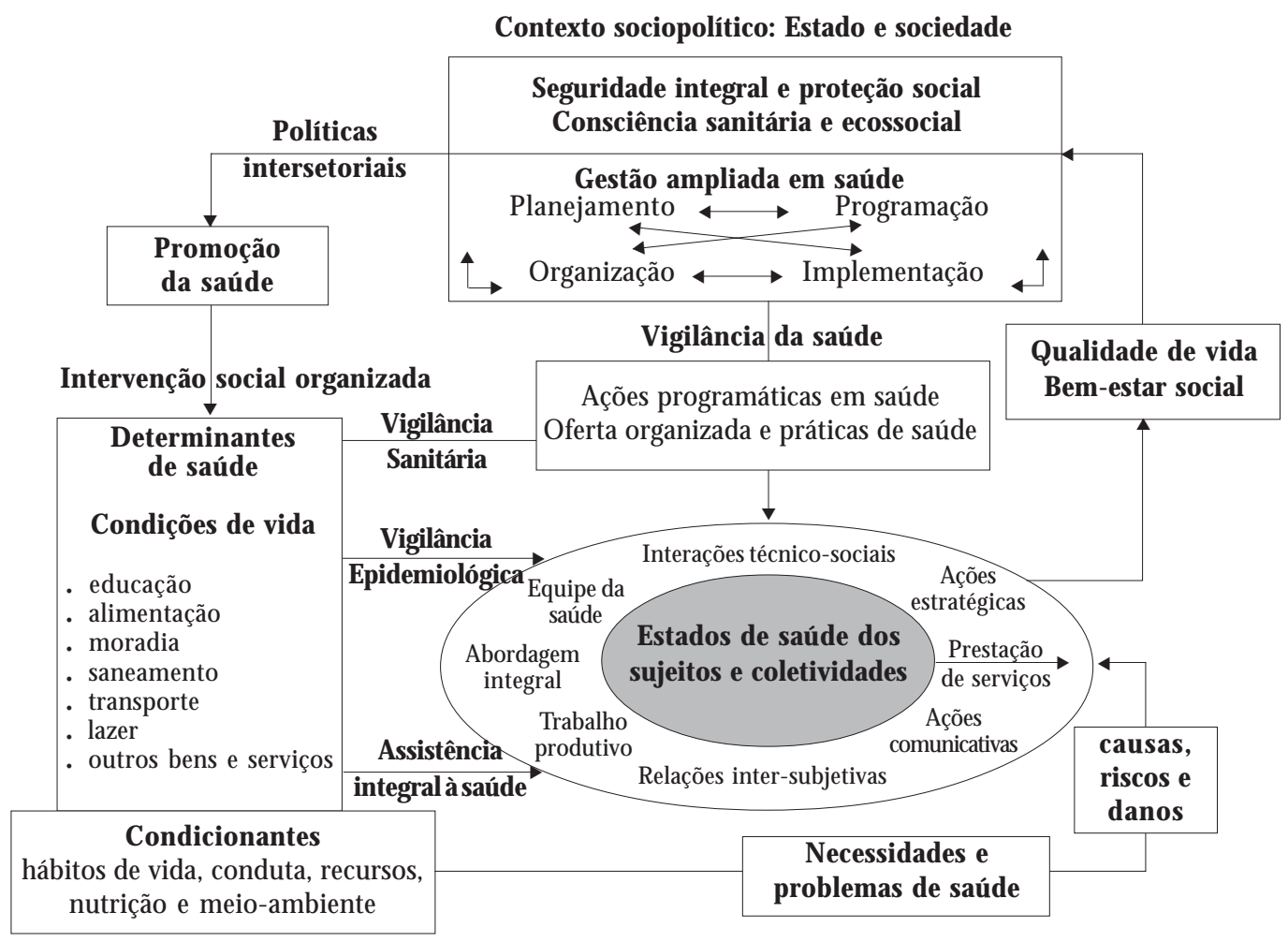

Figura 2. Contextualização dos níveis de atuação da vigilância da saúde sob uma lógica regionalizada e integralizada do Sistema Único de Saúde.

Adaptação: Paim²3.

menta imprescindível de gestão da saúde pública. Assim, a epidemiologia como saber tecnológico pode ser utilizada como instrumento para a elaboração depolíticas, planejamento local, definição de prioridades e avaliação em saúde, como na reorganização dos processos de trabal ho produtivo e reorientação programática das ações, tendo como base político-sanitária a vigilância e promoção da saúde ${ }^{29}$.

\section{Tendências}

\section{O campo dos saberes \\ e práticas da saúde coletiva brasileira}

Em princípio, compreendemos por Saúde Coletiva uma prática social de natureza transformadora para proteger e promover a saúde dos sujeitos e da coletividade, incluindo o compro- misso com a qualidade de vida ebem-estar social, bem como assegurar o acesso equitativo a cuidados integrais de saúdee a participação comunitária como peça fundamental da planificação eavaliação dos serviços. Logo, algumas de suas principais atribuições seriam a análise da situação de saúde segundo as condições de vida dos grupos sociais; a vigilância de causas, riscos e danos face ao processo saúde-doença; o fortalecimento da gestão ampliada e do planejamento participativo de saúde; a melhoria da qualidade na produção e prestação de bens e serviços equitativos e a pesquisa teórico-conceitual frenteà práxis da implementação de soluções criativas e inovadoras.

O campo da Saúde Coletiva abrange então o estudo das condições de saúde, a formulação de políticas públicas, a reorganização do processo de trabalho, como a avaliação das práticas e serviços de saúde. Como saber, articula-se em um eixo transinterdisciplinar, constituído pela epi- 
demiologia, planejamento e ciências sociais em saúde, com enfoque na geografia, história, sociologia, ciências políticas, dentreoutras. Enquanto prática, propõe mudanças no modelo assistencial de saúde, relevando uma consciência sanitária construtiva e participação social no processo de reorientação das políticas intersetoriais, tendo em vista a melhoria dos níveis de saúde e da qualidade de vida.

Já a promoção da saúde busca, a sua vez, modificar as condições de vida e saúde para que sejam dignas e equânimes e orienta-se ao conjunto de ações e decisões coletivas que possam favorecer a saúde, a qualidade de vida e o bemestar social; para esta, o objetivo práxico seria promover sempre melhores condições de vida e níveis de saúdemais satisfatório $\mathrm{s}^{30}$. Sob esse prisma, a concebemos como um processo de constituição de sujeitos e comunidades saudáveis para monitorar os determinantes ecossociais de saúde, incrementando os modos de andar a vida e modificando ou protegendo os meios da vida social. A presenta-se como uma estratégia de mediação entre sujeitos emeio social, combinando liberdade de escolha com responsabilidade social pela saúde, sendo que suas estratégias são interativas eintersetoriais, como supõe uma efetiva participação da coletividade desde sua formulação até sua implementação.

Freitas ${ }^{31}$, refletindo sobre a interface entre vigilância e promoção da saúde em nosso país, propõe que sejam formuladas políticas públicas saudáveis direcionadas para a criação de meios sociais favoráveis à saúde através da conquista da equidade, possibilitando novas alternativas deatuação sobreas desigualdades sociais em saúde, bem como orientando atitudes e condutas em direção ao pleno desenvolvimento integral da vida social humana, fortalecendo, também, os laços de solidariedade e poderes políticos das coletividades na legitimação dos processos decisórios democráticos frente às relações sociopolíticas e econômicas que afetam a saúde da socie dade como um todo.

Além disso, a proposta de territorialização da vigilância da saúde permite, de certo modo, reverter a lógica das decisões extraterritoriais e da regulação econômica, sendo vital para as ações de promoção da saúde orientadas tanto para o enfrentamento de doenças causadas pela exclusão social e pobreza e as geradas pelo processo iníquo de urbanização e industrialização em nosso país, quanto para a reorientação dos serviços de saúde em direção à superação das complexas e profundas desigualdades relativas à cobertura real, ao acesso equitativo, à oferta organizada e à qualidade ampliada na humanização do sistema nacional de saúde ${ }^{31}$.

A vigilância da saúde, ao apreender as iniquidades como um obstáculo aos plenos modos de andar a vida ou uma ameaça concreta ao bemestar social, poderia ajudar a promover ou proteger a saúde das pessoas ao contemplar os determinantes ecossociais de saúde, bem como reforçar o protagonismo dos sujeitos sobre as suas reais necessidades, para então intervir de modo progressivo sobre situações e não unicamente sobre riscos ${ }^{15}$.

Essas propostas guardam assim entre si estreitas afinidades, estando radicadas na mesma compreensão do que seja a tarefa de assistir à saúde e, portanto, de como apreender os seus objetos, meios e práticas. Contudo, ainda mais fortementequenesse modo tecnológico, aqui prevalece a concepção de que promoção da saúde não sedefineapenas pelo monitoramento ou controle daquilo que a pode ameaçar, pois a ampliação que esta postula é que almeja em intervir não apenas sobre o que se deve evitar para viver de forma saudável, mas quer também estabelecer patamares a serem atingidos em termos de aquisições positivas para produzir uma boa qualidade de vida do ponto de vista biopsicossocial ${ }^{32}$.

Devemos reconhecer ainda que as abordagens metodológicas em vigilância e promoção da saúde, por serem saberes e práticas mais recentes, todavia estão menos desenvolvidas que os métodos de planificação e organização dos programas de prevenção e controle de doenças. No entanto, ambas as estratégias são complementares enão excludentes no planejamento das ações e programas de saúde, sendo que a promoção afeta integralmente a todos os elementos que até agora vinham intervindo e, aportando novosinstrumentos, reorienta os arranjos tecnológicos e gerenciais de saúde pública ${ }^{30}$.

Por conseguinte, entendemos que, no âmbito da atenção integral à saúde, devem se realizar tanto as ações de proteção quanto as de promoção da saúde e de prevenção a riscos e danos, aí incluídos aqueles relativos à produção e consumo de bens e serviços de interesse à saúde da sociedade, como das suas interações com os meios de vida e trabalho produtivo, atribuições inerentes ao espaço de atuação multiprofissional da vigilância sanitária. Logo, os profissionais da atenção básica devem estar preparados para a identificação de riscos sanitários potenciais e danos à saúde da população do seu território, para agir de forma interdisciplinar e intersetorial, desen- 
volvendo tecnologias de cuidado no contexto da integralidade.

Aliás, buscar integralidade no SUS não seria apenas viabilizar o trabalho intersetorial nos diferentes níveis de atenção à saúde, mas articular as diversas ações locais, implementando um processo de trabalho sintonizado com as necessidades reais de saúde da coletividade atendida, em que as práticas da abordagem integral devam estar também dirigidas para cada encontro específico dos sujeitos com a equipe de saúde. Acrescenta-se ainda que a promoção da saúde, apresentando-seem todos os níveis de gestão do SUS, está comprometida com os serviços e ações que coloquem usuários e profissionais como protagonistas na organização do processo produtivo de saúde e na produção de sujeitos e seus modos de vida ${ }^{33}$.

Quanto à intersetorialidade, significaria um processo de construção compartilhada em que os diferentes setores envolvidos são tocados por saberes, práticas e modos de agir que lhes são usuais, pois pertencem ou se localizam no espaço de atuação dos seus parceiros institucionais, implicando a existência de algum grau de abertura em cada setor para dialogar e estabelecer vínculos de corresponsabilidade e cogestão pela me Ihoria da qualidade de vida da população. Porém, não deve ser pensada como uma estratégia única e ou definitiva a ser aplicada sobre os diferentes territórios e populações; pelo contrário, deve responder às necessidades de saúde de uma coletividade, mobilizando-a e envolvendo-a na elaboração das ações intersetoriais a serem implantadas ${ }^{33}$.

N esse sentido, a VS constitui-se de fato num esforço para integrar a atuação dos setores de saúde sobre as múltiplas e complexas dimensões do processo saúde-doença, especialmente do ponto de vista de sua determinação ecossocial e, a partir daí, buscar desenvolver novas formas de operacional ização para os sistemas e serviços de saúde, relevando assim uma visão que se pretende mais equânime e totalizadora da vida social humana. Nesse novo olhar e modos de agir em saúde, levam-se em conta os múltiplos processos envolvidos na gênese, no desenrolar ena perpetuação dos problemas de saúde, propondo a interação e envolvimento de todos os setores e atores inseridos naquela realidade e vendo os sujeitos e a comunidade como parte desse processo social, político e histórico ${ }^{34}$.

Ademais, o trabalho em saúde, estando imerso no contexto territorial, consegue definir os problemas e suas prioridades de modo mais ar- ticulado e integrado, como obter os recursos suficientes para atender as necessidades sociais de saúde conforme cada situação específica de diferentes coletividades. Onde território éconcebido como um espaço de coexistência entre grupos sociais com suas condições de vida e trabalho e acesso à educação, sendo parte do meio social da vida de comunidades saudáveis, de políticas públicas e de concepções sobre a saúde e doença, expressando uma cultura e saberes vivenciados. Portanto, toda equalquer ação que se realize deverá incidir sobre os indivíduos, famílias, comunidades e meios sociais, uma vez que promover a saúde e melhorar a qualidade de vida das pessoas implica o saber agir no contexto em queelas se inserem e nos espaços onde vivem.

Abrindo parênteses, refletimosqueosindivíduos, coexistindo em territórios socialmenteconstituídos, organizam-se em coletividades, ao longo do tempo, conformando uma história de caminhos traçados por onde percorreram o seu futuro. Logo, a sociedade como coletivos humanos se constitui numa totalidade-sujeito tendo por referência qualidades epistêmicas que a estruturam; as singularidades afloradas se conservam e superam na totalidade maior que as engloba ${ }^{35}$. Diríamos ainda que a ação autônoma nos meios da vida social supõe agir de forma responsável na organização política edestinos de uma comunidade, que deriva da liberdade de escolha e poder transformador na criação de formas equitativas de vida que promovam a autorrealização no âmbito da interação social, numa ordem em que a reflexividade interliga o sujeito aos sistemas da modernidade tardia ${ }^{36}$.

Diante do exposto, apesar de promissora a proposta de promover a saúde, é ao mesmo tempo desafiadora e complexa, tanto do ponto de vista político quanto do técnico-sanitário, implicando o estabelecimento de agendas públicas e ações intersetoriais com a participação de diversos atores e organizações, envolvendo também pessoas e comunidades para se alçar mais saúdee uma melhor qualidade de vida. Muitas das políticas e mobilizações sociais necessitam, para o seu triunfar, de uma abrangência e compromissosque extrapolam o território municipal e/ou regional, tornando-se necessário que se discutam novas formas de relações sociopolíticas en treas instituições do Estado e sociedade para que atendam melhor as necessidades de saúde dos grupos mais vulneráveis às fragilidades da vida, enão apenas à assistência de seus riscos, agravos e ou doenças ${ }^{34}$.

Já no processo de construção de sociedades saudáveis, se faz fundamental o exercício da ci- 
dadania, de tal modo que toda a coletividade possa ver seus principais direitos concretizados no seio do bem comum do sujeito social da vida, necessitando, todavia, de meios estratégicos de seguridade integral, gestão sanitária e consciência ecossocial. Sendo preciso definir, ainda, as possibilidades e dificuldades dentre todos os envolvidos para se estabelecer compromissos epactuá-los politicamente, a partir de um diálogo em que os atores envolvidos se disponham a reconhecer e legitimar a força de atuação uns dos outros e não uma relação contraditória de domínio e imposição de um ator sobre o outro.

Lembrando quea interface en tre gestão esubjetividade corresponde ao entendimento da organização como coletivo que aprende edo plane jamento como comunicação voltada para a re flexão crítica dos vários enfoques desdeuma perspectiva situacional e comunicativa; em que o diálogo, argumentação, visão de futuro e capacidade de mudar produzem saberes e práticas comuns a todos que recondicionam a cultura organizacional e a intersubjetividade ali inserida, como possibilidades de uma gestão ampliada, democrática e equânime de saúde ${ }^{37}$.

Retornando à concepção de vigilância da saúde sobre a prevenção dos riscos de adoecer, entendemos que as análises das condições de vida, como da reprodução social e sobrevivência, estão igualmente voltadas para apreender os riscos edanos frente aos grupos sociais imersos em seus territórios e modos de andar a vida. A primazia desse processo está em estabelecer novos elos causais e soluções que podem sobrepujar 0 atendimento pontual e fragmentado dos problemas da saúde. D onde os serviços de VE e sanitária, de saúde ambiental e programas de saúde devem procurar novas interfaces e trabalhar de modo integrado na detecção e controle dos múltiplos riscos relacionados ao processo saúde-doença. As ações programáticas de saúde necessitam assim ser repensadas, no sentido do desenvolvimento de intervenções para as situações de risco social, sanitário e ambiental, como da efetiva re dução dos inúmeros agravos vinculados a essas condições epidemiológicas ${ }^{34}$.

Não obstante de uma prática de integralidade das ações, deve-se a priori concretizar um trabal ho em equipe que estabel eça estratégias de diálogo transdisciplinar dentre os núcleos de cada prática profissional e campos de ação da Saúde Coletiva, contribuindo para promover a saúde tanto no âmbito individual quanto coletivo. A noção de cuidado integrado compreende, pois, os modos de agir dos diferentes atores na pro- dução de saúde e sujeitos produtores, em que 0 cuidar degentese constitui em espaços de escuta, acolhimento, relação ética e diálogo entre os envolvidos na produção do cuidado e seu valor de uso. A integralidade como um princípio norteador do SUS deve orientar a atenção à saúde de modo $a$ atender às necessidades reais e demandas potenciais dos sujeitos, grupos e coletividades, num novo paradigma para a saúde pública em sua práxis do saber agir ${ }^{38}$.

Com base nesse princípio edo agir em saúde, os serviços devem oferecer então ações de promoção à saúde, proteção e prevenção de riscos e danos, como recuperação e reabilitação dos sujeitos adoecidos em face à dinâmica do processo saúde- doença, sendo que estas devem estar articuladas e integradas em todos os espaços de atuação do sistema nacional de saúde. É nesse contexto que se faz presente um novo modo de intervenção voltado para a articulação entre a assistência e as ações de Saúde Coletiva, buscando superar a dissociação já cristalizada entre os serviços de saúde pública e de atenção primária e médico-hospitalar.

Já no quetangeà prática de educação em saúde, como um caminho integrador do cuidar, se constitui em um espaço de reflexão para a ação capaz de provocar mudanças nos sujeitos e prontidão para atuar na família e na comunidade, intervindo no controlee implementação depolíticas públicas para a transformação dos modos de andar a vida com saúde. 0 exercício de uma prática educativa crítica, como práxis da realidade humana social, se constitui assim em um modo de interven ção sobre a vida, rejeitando qualquer forma de exclusão ou dominação e integrando uma atitude reconstrutiva para se viver melhor e cuidar da saúde ${ }^{38}$.

Logo, a construção de um processo educativo em saúde, que atenda ao princípio norteador da integralidade, requer a participação ativa das pessoas e coletividades na leitura e reflexão crítica de sua realidade social em busca de condições mais dignas de vida, como um sujeito histórico do saber agir, permeado por suas descobertas e inquietações frente ao modo de viver em sociedade e exercendo sua cidadania para se emancipar e cuidar melhor da saúde.

Em tese, podemos argumentar que todo 0 arcabouço conceitual-operacional de vigilância da saúde se constitui como uma base política e sanitária para a consolidação do paradigma da promoção da saúde no campo da Saúde Coletiva. Logo, se faz necessário resgatar os horizontes filosóficos que a proposta traz para o modo 
como as práticas de saúde devam ser organizadas e implementadas, para além e/ou por meio de suas finalidades técnicas, gerando processos criativos e solidários de melhoria da vida e saúde dos indivíduos e coletividades.

Sem a reflexão, não há como dar conta do desafio detraduzir informações geradas por meio da produção científica em ações que possam efetivamente promover transformações sociais, ambientais e de condutas não saudáveis dos sujeitos. Os desafios que se colocam, nesse sentido, não se resolvem somente com a aplicação de no-

\section{Colaboradores}

ALV Arreaza e JC de M oraes participaram igualmente de todas as etapas da elaboração do artigo. vos model os conceituais, da mesma maneira que a reconfiguração das práticas de saúde não se resolve somente com a informação e capacitação técnica dos agentes envolvidos.

Por fim, parafraseando Barreto ${ }^{39}$, na tensão entre saber e ação, a epidemiologia traz à tona para seus praticantes os devires dialéticos dentre "sonhar efazer; utopia e realidade; técnica e política" e, acrescente-se, "saberes epráticas; social e sanitário; sujeito e estrutura; promoção e prevenção; coletivo e individual; ou ainda, saúde e doença".

\section{Referências}

1. Waldman EA. Usos da vigilância e da monitorização em saúde pública. IESUS 1998; 7(3):7-26.

2. Langmuir $A D$. The surveillance of communicable diseases of national importance. NEJM 1963; 268(4):182-192.

3. Declich S, Carter AO. Public health surveillance: historical origins, methods and evaluation. WHO Bulletin 1994; 72(2):285-304.

4. Raska K. National and international surveillance of communicable diseases. WHO Chron 1966; 20:315321.

5. Costa EA, Rozenfeld SA. A constituição da vigilância sanitária no Brasil. In: Rozenfeld SA, organizador. Fundamentos da vigilância sanitária. Rio de Janeiro: Fiocruz; 2000.

6. Costa EA. A vigilância sanitária: defesa e proteção da saúde [tese]. São Paulo (SP): Faculdade de Saúde Pública/USP; 1998.

7. Thacker SB, Berdelman RL. Public health surveillance in the United States. Epidemiol Rev 1988; 10:164-190.

8. Thacker SB, Stroup DF, Parrish RG, Anderson HA. Surveillance in environmental public health: issues, systems and sources. Am J Public Health 1996; 86(5):633-638.

9. Waldman EA. A vigilância como instrumento de saúde pública. In: Vecina-N eto G, Cutait R, Terra V, organizadores. Vigilância em Saúde Pública. São Paulo: Fundação Peirópolis; 1998. p. 91-131.

10. Teixeira CF, Paim JS, Vilasbôas AL. SUS, modelos assistenciais e vigilância da saúde. IESUS 1998; 7(2):7-28.

11. Barata RB, Barreto ML. Algumas questões sobre 0 desenvolvimento da epidemiologia na América Latina. Cien Saude Colet 1996; 1(1):70-79.

12. Pereira MG. Epidemiologia: teoria e prática. $5^{a} \mathrm{ed}$. Rio de Janeiro: Guanabara Koogan; 2001. p. 469-71. 
13. Paim JS, Teixeira M GLC. Reorganização do sistema de vigilância epidemiológica na perspectiva do Sistema Ú nico de Saúde. In: 10 Seminário Nacional de Vigilância Epidemiológica; 1992; Brasília. p. 93-144.

14. Drumond Jr. M. Epidemiologia nos municípios: muito além das normas. São Paulo: Hucitec; 2003. p. 5366.

15. Paim JS. Vigilância da Saúde: dos modelos assistenciais para a promoção da saúde. In: Czeresnia D, Freitas CM , organizadores. Promoção da Saúde: conceitos, reflexões e tendências. Rio de Janeiro: Fiocruz; 2003. p. 161-174.

16. Fundação Nacional de Saúde. Guia de Vigilância Epidemiológica. 5a ed. Brasília: M inistério da Saúde; 1998.

17. Mendes ACG, Silva Jr. JB, M edeiros KR, Lyra TM, Melo Filho DA, Sá DA. Avaliação do Sistema de Informações Hospitalares: como fonte complementar na vigilância e monitoramento de doenças de notificação compulsória. IESU S 2000; 9(2):67-86.

18. Teixeira MGLC, Penna GO, Risi JB, Penna ML, Alvim MF, Moraes JC, Luna E. Seleção das doenças de notificação compulsória: critérios e recomendações para as três esferas de governos. IESU S 1998; 7(1):7-28.

19. Penna MLF. Epidemiologia e serviços de saúde: momento atual. In: 10 Seminário Nacional de Vigilância Epidemiológica; 1992; Brasília. p. 53-56.

20. Forattini OP. Ecologia, epidemiologia e sociedade. $2^{\underline{a}}$ ed. São Paulo: Artes M édicas; 2004. p. 405-408.

21. Secretaria de Vigilância em Saúde. Guia de vigilância epidemiológica. 6a ed. Brasília: M inistério da Saúde; 2005.
22. Castellanos PL. Epidemiología y organización de los servicios. In: OPS/OMS, organizador. La formación en epidemiología para el desarrollo de los servicios de salud. Washington, D.C.: OPS/OM S; 1987. p. 30-40. [Serie Desarrollo de Recursos Humanos no 88]

23. Paim JS. A reforma sanitária e os modelos assistenciais. In: Rouquayrol MZ, Almeida-Filho N, organizadores. Epidemiologia e Saúde. 5a ed. Rio de Janeiro: M edsi; 1999. p. 473-487.

24. Mendonça EF, Cosenza GW. Repensando a vigilância epidemiológica. In: Costa M F, Souza RP, organizadores. Qualidade de vida: compromisso histórico da epidemiologia. Belo Horizonte: Coopmeed/Abrasco; 1994. p. 277-280.

25. Relatório de Oficina de Trabalho. Vigilância à Saúde. In: III Congresso Brasileiro de Epidemiologia; 1997; Salvador.

26. M endes EV. Distrito Sanitário: o processo social de mudança das práticas sanitárias do Sistema Único de Saúde. São Paulo: Hucitec; Rio de Janeiro: Abrasco; 1993.

27. Teixeira CF. Epidemiologia e planejamento de saúde. Cien Saude Colet 1999; 4(2):287-303.

28. Rivera FJU, Artmann E. Planejamento e gestão em saúde: flexibilidade metodológica e agir comunicativo. Cien Saude Colet 1999; 4(2):355-365.

29. Paim JS. Epidemiologia e planejamento: recomposição das práticas epidemiológicas na gestão do SUS. Cien Saude Colet 2003; 8(2):557-567.

30. Buss PM. U ma introdução ao conceito de promoção da saúde. In: Czeresnia D, Freitas CM, organizadores. Promoção da Saúde: conceitos, reflexões e tendências. Rio de Janeiro: Fiocruz; 2003. p. 15-38.

31. Freitas CM . A vigilância da saúde para a promoção da saúde. In: Czeresnia D, Freitas CM, organizadores. Promoção da Saúde: conceitos, reflexões e tendências. Rio de Janeiro: Fiocruz; 2003. p. 141-159. 
32. Ayres JR. Norma e formação: horizontes filosóficos para as práticas de avaliação no contexto da promoção da saúde. Cien Saude Colet 2004; 9(3):583-592.

33. Campos GWS, Barros RB, Castro AM . Avaliação de política nacional de promoção da saúde. Opinião. Cien Saude Colet 2004; 9(3):745-749.

34. Campos CEA. O desafio da integralidade segundo as perspectivas da vigilância da saúde e da saúde da família. Cien Saude Colet 2003; 8(2):569-584.

35. Barata RB. Epidemiologia no século XXI: perspectivas para o Brasil. Rev. bras. epidemiol. 1999; 2(1/ 2):6-18.

36. Giddens A. M odernidade e identidade. Rio de Janeiro: Jorge Zahar Editor; 2002. p. 193-212.

37. U ribe FJ. Reflexões sobre a subjetividade na gestão a partir do paradigma da organização que aprende. Cien Saude Colet 2001; 6(1):209-219.

38. Machado MFA, Monteiro EM L, Queiroz DT, Vieira NFC, Barroso M GT. Integralidade, formação de saúde, educação em saúde e as propostas do SUS: uma revisão conceitual. Cien Saude Colet 2007; 12(2):335-342.

39. Barreto ML. Por uma epidemiologia de saúde coletiva. Rev. bras. epidemiol. 1998; 1(2):104-122.

Artigo apresentado em 19/11/2007

Aprovado em 28/042008 